\title{
Research Article \\ Classification of Hallucal Sesamoid Bone Correlated with Hallux Valgus Severity
}

\author{
Lei Zhang $\mathbb{D}^{1,2,3,4}$ Junqiu Wang, ${ }^{2,5}$ Jiaju Liu, $^{6}$ and Jiangqin Luo $\mathbb{D}^{7}$ \\ ${ }^{1}$ Department of Orthopedics, Affiliated Traditional Chinese Medicine Hospital of Southwest Medical University, Luzhou, China \\ 646000 \\ ${ }^{2}$ Academician Workstation in Luzhou, Luzhou, China 646000 \\ ${ }^{3}$ Center for Orthopedic Diseases Research, Affiliated Traditional Chinese Medicine Hospital of Southwest Medical University, Luzhou, \\ China 646000 \\ ${ }^{4}$ Clinical Base of Affiliated Traditional Chinese Medicine Hospital of Southwest Medical University, Guangdong Province Medical 3D \\ Printing Application Transformation Engineering Technology Research Center, Guangdong Province, China 510000 \\ ${ }^{5}$ School of Chinese and Western Clinical Medicine, Southwest Medical University, Luzhou, China 646000 \\ ${ }^{6}$ Market Supervision Administration of Luzhou City of Sichuan Province, Kangcheng Road, Jiangyang District, Luzhou City, \\ Sichuan Province, China 646000 \\ ${ }^{7}$ Department of Operation Theatre, Affiliated Traditional Chinese Medicine Hospital of Southwest Medical University, Luzhou, \\ China 646000
}

Correspondence should be addressed to Jiangqin Luo; 913403858@qq.com

Received 17 February 2020; Accepted 20 May 2020; Published 27 June 2020

Academic Editor: Sae Hoon Kim

Copyright (C) 2020 Lei Zhang et al. This is an open access article distributed under the Creative Commons Attribution License, which permits unrestricted use, distribution, and reproduction in any medium, provided the original work is properly cited.

\begin{abstract}
The hallucal sesamoid bones (HSBs), having an important role in reducing load per unit area on the first metatarsal head, can be injured commonly which also affected the first metatarsophalangeal joint and the surrounding structure. Meanwhile, differences among each HSB type may be a major factor affecting the occurrence and development of HV. So far, many researchers had learned that there are three different conditions in hallucal sesamoid bone affecting the choice of clinical surgery corresponding to different solutions in clinic. Thus, it is necessary to study the anatomical morphological characteristics of the HSB which can be helpful in clinical diagnosis and treatment, especially hallux valgus (HV). $150 \mathrm{X}$-ray and three-dimensional (3D) computed tomographic (CT) images consist of 72 left and 78 right metatarsals were applied in this anatomic study between two variables and showed by a simple scatter plot. The first metatarsophalangeal joint is divided into four different types: type I (no HSB, 1.3\%), type II (with one HSB, 0.07\%), type IIIa (with two HSBs when THB is bigger, 28\%), type IIIb (with two HSBs when FHB is bigger, 65.3\%), and type IV (with three HSBs, 4.7\%). There was no statistical difference between the left and right sides, except HVA, Meary, and pitch $(P<0.05)$; all a, b, c, d, and i have statistical difference between male and female $(P<0.05)$. Meanwhile, HVA and IMA and HVA and type group have a significant correlation. In summary, HVA and IMA and HVA and classification of HSBs have significant correlations. The classification and location of HSBs can be an important basis to choose operation methods and postoperation evaluation.
\end{abstract}

\section{Introduction}

Hallux valgus (HV) is a complex anatomical deformity that is challenging in treatment. Park et al. [1] pointed out that HV is a highly prevalent foot deformity that involved hallucal sesamoid bones (HSBs) and affected $23 \%$ of adults and $35.7 \%$ of elderly individuals. In the normal walking gait of a man, the hallucal sesamoid complex transmits 50\% weight of the body and can transmit over $300 \%$ load during push-off, when the tibial side one undertakes the most part. That means HSBs play an important role in reducing load per unit area in the first metatarsal head, especially the tibial side one. Clinically, patients with sesamoid bones below the first metatarsal head were three to five times more likely to suffer HV than patients 
without sesamoid bone [2-5]. That means the classification of sesamoids may be a major factor affecting the occurrence and development of HV [6]. From this, it is necessary to learn about the relationship between classification of HSBs and HV severity which can be helpful to assist in clinical treatment and providing data indexes for postoperation evaluation.

In 1986, Mann and Coughlin [7] gave the first description of the severity of HV named Mann Type which included mild, moderate, and severe. The measurement data of HV are from weight-bearing X-rays. The imaging retrospective study of 500 cases of $\mathrm{HV}$ and 500 cases of non-HV foot in Theumann et al. [6] showed that the incidence of multiple seed bone in HV foot (32.3\%) was significantly higher than that in non-HV foot (15.2\%). Munuera et al. [8] found that there were significant differences between the $\mathrm{HV}$ angle of the multiseeded and nonfractionated feet by 474 foot imaging studies. Usually, there are two sesamoid below the head of the first metatarsal, the inner side one named tibial hallux sesamoid (THS) and the offside one named fibular hallux sesamoid (FHS); besides, THS is bigger than FHS [9-11]. However, all differences are only for the tibial bone, and the causal relationship between HSBs and HV is not clear now. Meanwhile, the "capstan mechanism" that exists in the first metatarsophalangeal joint will aggravate the first metatarsal varus and aggravate the relative subluxation of HSBs. So each classification of Mann Type touches upon HSBs subluxated, dislocation and a larger proximal articular set angle are obvious indicators for clinical operation [12-15]. To complete the data acquisition, a fitful method to measure the dislocation of seed bone is needed. By comparing, Hardy classification is the most incredible and rich method to tell the dislocation of seed bone [16]. However, Grade IV classification, which is based on Hardy classification, is more simple, practical, and fit for this research [17]. Clinically, the best choice for the treatment of HV is surgery. Different conditions always match with different solutions; surgical options for managing these problems include curettage, bone grafting, shaving, internal fixation, and partial or complete excision [18-21]. Thus, surgeons need to develop the best treatment plan through more data references. Anatomically, the sesamoid bone located at the muscle insertion between the tendon and the bone and is formed by tenostosis can change the angle of tendon insertion, enlarge the muscle power, and increase the muscular contractility. The stability of sesamoid is maintained by the ligament between them, the thickness of them, and the attachment of tendon. When the muscle group around them is stacked into power imbalance because of the surgery, worse pathology comes. Hence, it is necessary to learn the classification and location of HSBs, so that the relationship between the classification of HSBs and HV severity can be analyzed. The results can be an important basis to remind patients of prevention, help surgeons choose operation methods, and do the postoperation evaluation.

In general, the choice of HV surgery is the basic skill that the ankle surgeon needs to master. This study illustrated the relationship between the classification of HSB and HV severity to provide auxiliary parameters for ankle surgeons to

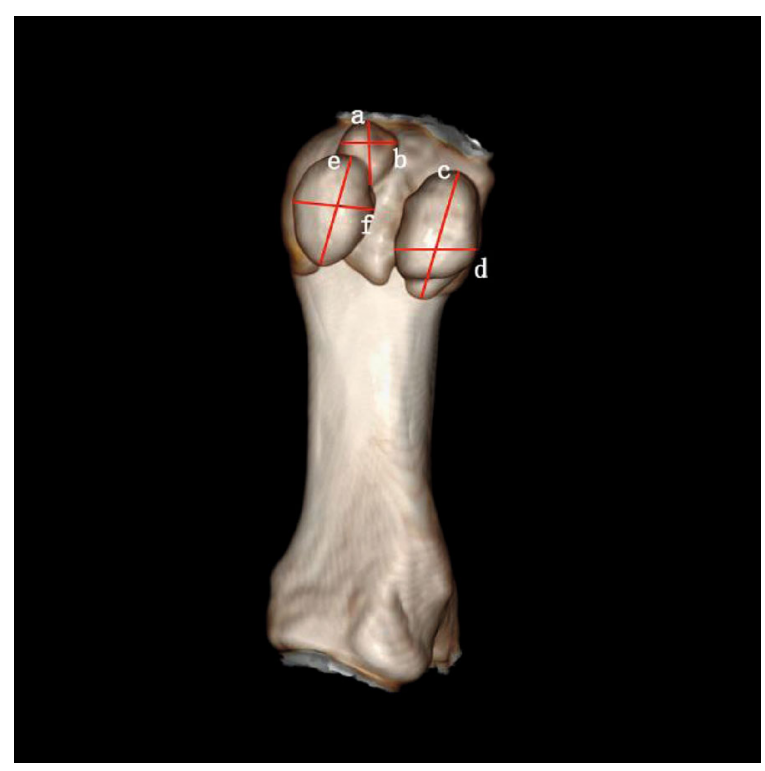

FIgURE 1: The long and short axis of the sesamoid bone. (a) The long axis of the medial distal sesamoid bone (the longest distance of the medial distal sesamoid bone). (b) The short axis of the medial distal sesamoid bone (the shortest distance of the medial distal sesamoid bone). (c) The long axis of the lateral distal sesamoid bone. (d) The short axis of the lateral distal sesamoid bone. (e) The long axis of the medial proximal sesamoid bone. (f) The short axis of the medial proximal sesamoid bone.

select suitable surgical methods for patients. We measured 302 HSBs from 150 dry metatarsals from the Chinese to master the basic anatomical structure and morphology of the Chinese HSBs, which can improve the anatomical data of it and be helpful in clinical diagnosis and treatment.

\section{Material and Methods}

2.1. Materials. $302 \mathrm{HSBs}$ from 150 metatarsals which consist of 72 left and 78 right metatarsals were measured by X-ray and three-dimensional (3D) computed tomographic (CT) reconstruction techniques, which were weight-bearing, and recorded with an accuracy of up to $0.1 \mathrm{~mm}$. All the 100 male and 50 female (mean 41 years, range 18-90 years) metatarsals belong to the Chinese Han nationality supposed by the Ethical Inspection Committee at Southwest Medical University (Luzhou, China).

2.2. Instruments. The $\mathrm{X}$-ray and three-dimensional (3D) computed tomographic (CT) data of metatarsal were collected from digital radiography and a spiral CT scanner (Somatom Emotion; Siemens AG, Munich, Germany). Measurements were carried out by the software (UniReport version 2.0) of the PACS. Both the X-ray and CT scans were weight-bearing.

\subsection{Method of Measure}

(1) a: the long axis of the medial distal sesamoid bone (the longest distance of the medial distal sesamoid bone) 


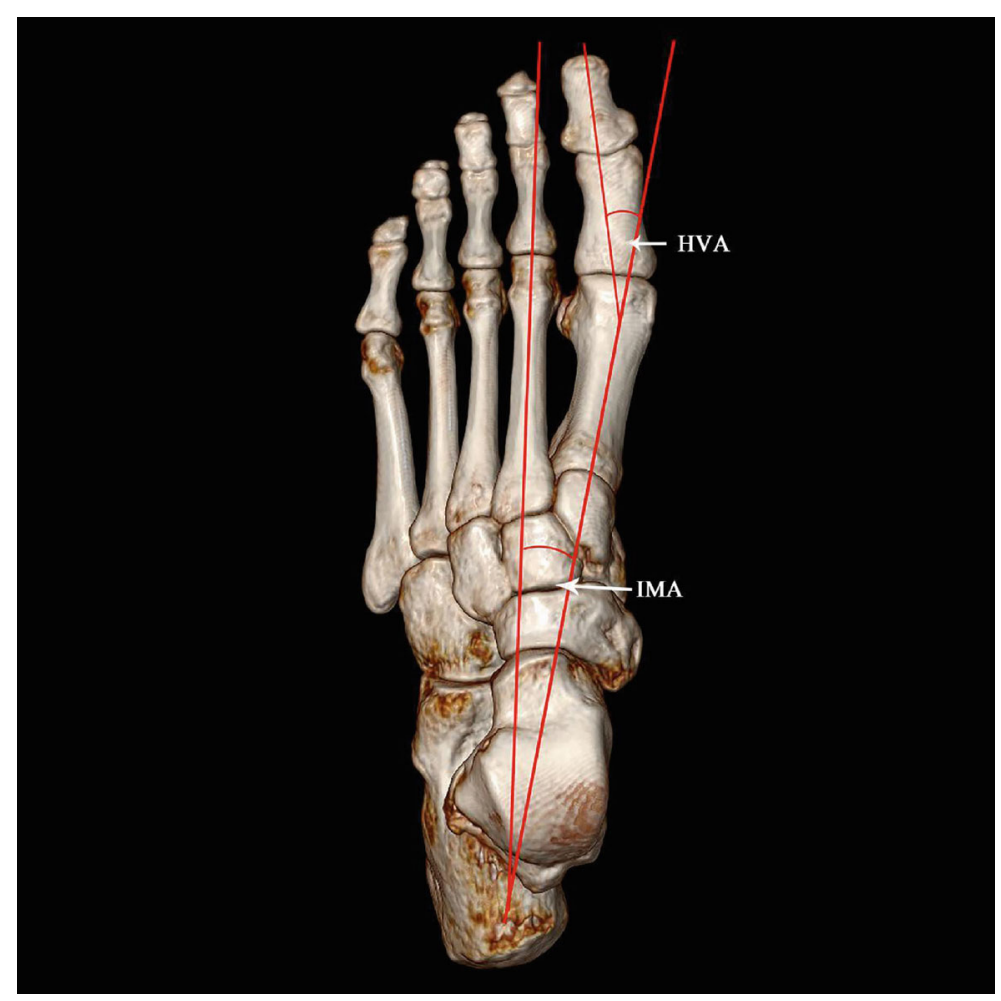

Figure 2: The angle to judge the severity of HV. On orthographic X-rays, the angle between the longitudinal axis of the first metatarsal bone and the longitudinal axis of the first phalangeal proximal phalangeal bone, normal $<16^{\circ}$. Intermetatarsal IMA: on the orthographic X-ray film, the angle between the longitudinal axis extension line of the first metatarsal bone and the second metatarsal bone, normal $<10^{\circ}$.

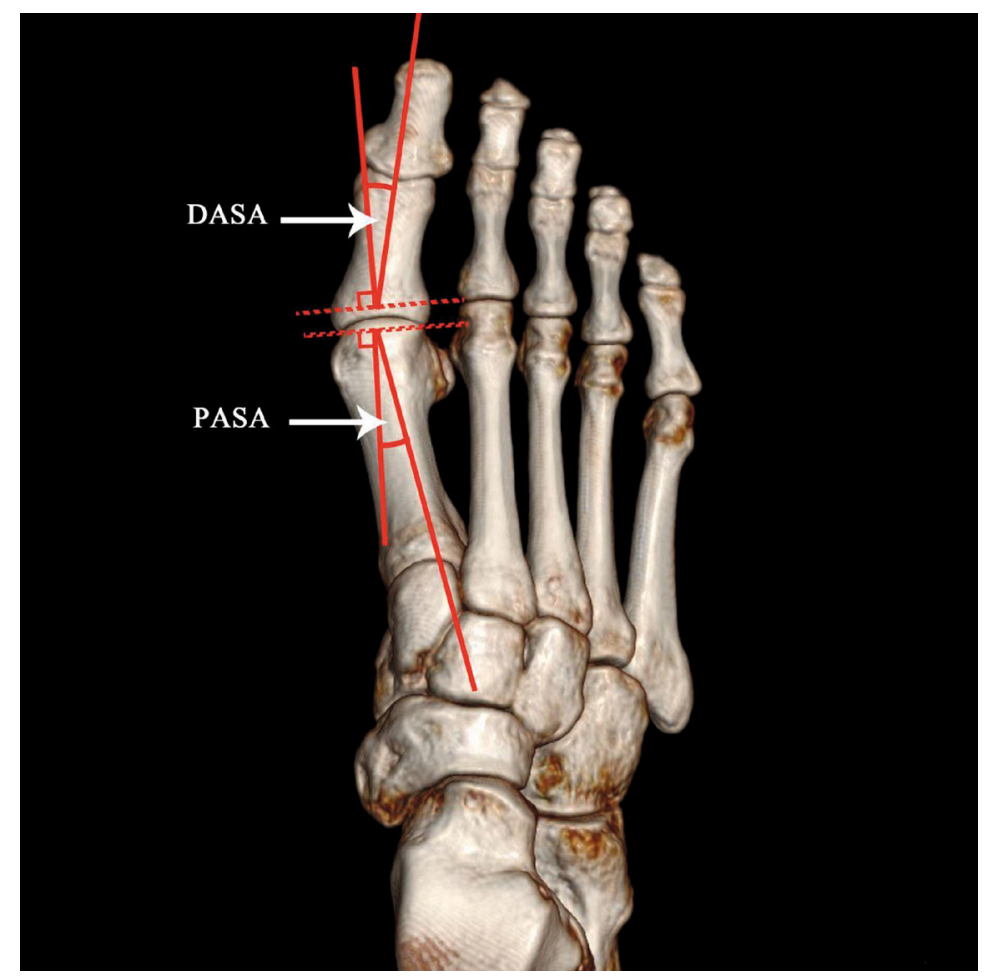

Figure 3: The angle to assist in judging the severity of HV. Distal articular set angle (DASA): on orthographic X-rays, the angle between the vertical line of the basal joint surface of the first phalangeal bone and the long axis of the first phalangeal bone, normal $<7^{\circ}$. Proximal articular set angle (PASA): on the orthographic X-ray film, the angle between the vertical line of the first metatarsal joint and the first metatarsal axis, normal $<10^{\circ}$. 


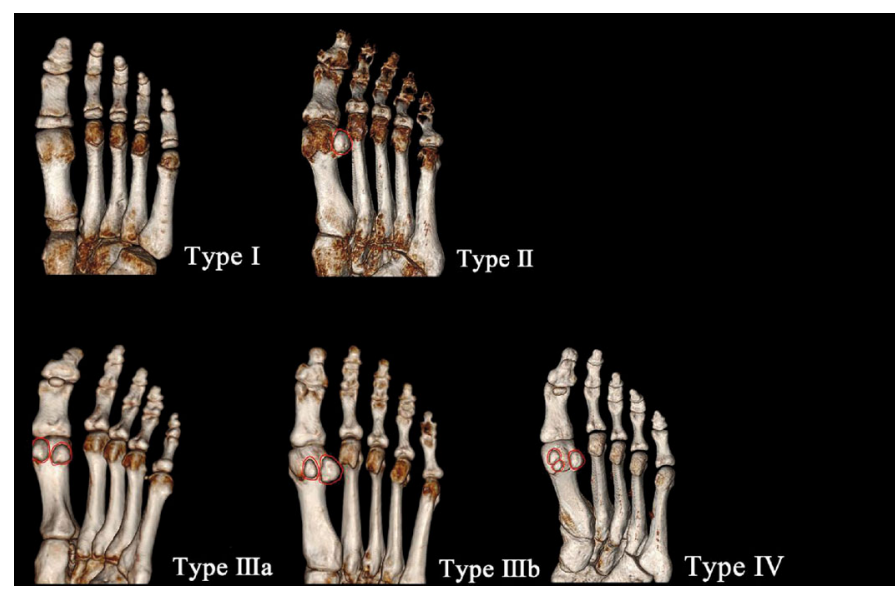

FIgure 4: The types of HSBs on inferior by 3D CT image. Type I: no HSB; type II: with one HSB; type IIIa: with two HSBs when THB is bigger; type IIIb: with two HSBs when FHB is bigger; and type IV: with three HSBs. All HSBs are circled in red.

TABLE 1: Frequency of HVA and IMA severity in each classification found in 3D CT images.

\begin{tabular}{lcccccc}
\hline & Mild & $\begin{array}{r}\text { HVA }\left(^{\circ}\right) \\
\text { Moderate }\end{array}$ & Severe & Mild & $\begin{array}{r}\text { IMA }\left(^{\circ}\right) \\
\text { Moderate }\end{array}$ & Severe \\
\hline I & 0 & 0 & 0 & 0 & 0 & 0 \\
II & 0 & 1 & 0 & 1 & 0 & 0 \\
IIIa & 40 & 2 & 0 & 41 & 1 & 0 \\
IIIb & 94 & 4 & 0 & 95 & 3 & 0 \\
IV & 5 & 2 & 0 & 7 & 0 & 0 \\
\hline
\end{tabular}

Note: mild (grade) in HVA is less than 25 degrees, moderate (grade) in HVA is between 25 and 35 degrees, and severe (grade) in HVA is more than 35 degrees; mild (grade) in IMA is less than 15 degrees, moderate (grade) in IMA is between 15 and 20 degrees, and severe (grade) in IMA is more than 20 degrees.

(2) b: the short axis of the medial distal sesamoid bone (the shortest distance of the medial distal sesamoid bone)

(3) c: the long axis of the lateral distal sesamoid bone (the longest distance of the lateral distal sesamoid bone)

(4) d: the short axis of the lateral distal sesamoid bone (the shortest distance of the lateral distal sesamoid bone)

(5) e: the long axis of the medial proximal sesamoid bone (the longest distance of the medial proximal sesamoid bone)

(6) f: the short axis of the medial proximal sesamoid bone (the shortest distance of the medial proximal sesamoid bone)

(7) i: distance between medial and lateral sesamoid bones (Figure 1)

(8) Degree of dislocation in sesamoid bone: taking the bisection line of the long axis of the first metatarsal axis as the reference line. Grade 0: no displacement of medial sesamoid relative to reference
TABLE 2: Frequency of medial HSB displace grade found in 3D CT images.

\begin{tabular}{cccccc}
\hline & & \multicolumn{4}{c}{ Dislocation* } \\
& & Grade 0 & Grade 1 & Grade 2 & Grade 3 \\
\hline \multirow{5}{*}{ Classification } & I & - & - & - & - \\
& II & - & - & - & 1 \\
& IIIa & 10 & 19 & 12 & 1 \\
& IIIb & 31 & 36 & 29 & 2 \\
& IV & 3 & 2 & 2 & - \\
\hline
\end{tabular}

$* P<0.05$ vs. classification. Note: taking the bisection line of the long axis of the first metatarsal axis as the reference line: Grade 0: no displacement of sesamoid relative to the reference line; Grade 1: overlap of less than $50 \%$ of sesamoid to the reference line; Grade 2: overlap of greater than $50 \%$ of sesamoid to the reference line; and Grade 3: complete displacement of medial sesamoid beyond the reference line laterally.

line; Grade 1: overlap of less than $50 \%$ of medial sesamoid to reference line; Grade 2: overlap of greater than $50 \%$ of medial sesamoid to reference line; Grade 3: complete displacement of medial sesamoid beyond reference line laterally

(9) Hallux valgus angle (HVA): on orthographic X-rays, the angle between the longitudinal axis of the first metatarsal bone and the longitudinal axis of the first phalangeal proximal phalangeal bone, normal $<16^{\circ}$

(10) Intermetatarsal angle (IMA): on the orthographic $\mathrm{X}$-ray film, the angle between the longitudinal axis extension line of the first metatarsal bone and the second metatarsal bone, normal $<10^{\circ}$ (Figure 2)

(11) Proximal articular set angle (PASA): the angle between the vertical line of the first metatarsal joint and the first metatarsal axis, normal $<10^{\circ}$

(12) Distal articular set angle (DASA): on orthographic $\mathrm{X}$-rays, the angle between the vertical line of the basal joint surface of the first phalangeal bone and the long axis of the first phalangeal bone, normal $<7^{\circ}$ (Figure 3) 
TABLE 3: Mean values of measurements of sesamoid bones and the first metatarsophalangeal joint (mean \pm SD).

\begin{tabular}{|c|c|c|c|c|c|}
\hline & Term & Total & Left & Right & $P$ value \\
\hline \multirow{7}{*}{ Distance $(\mathrm{mm})$} & $\mathrm{a}$ & $11.765 \pm 1.905$ & $12.083 \pm 1.854$ & $11.465 \pm 1.916$ & 0.324 \\
\hline & $\mathrm{b}$ & $8.198 \pm 1.354$ & $8.117 \pm 1.311$ & $8.269 \pm 1.395$ & 0.391 \\
\hline & c & $11.882 \pm 1.361$ & $12.106 \pm 1.145$ & $11.692 \pm 1.501$ & 0.205 \\
\hline & $\mathrm{d}$ & $8.948 \pm 1.132$ & $9.059 \pm 1.198$ & $8.851 \pm 1.070$ & 0.379 \\
\hline & e & $8.478 \pm 2.646$ & $9.350 \pm 2.224$ & $7.925 \pm 3.352$ & 0.590 \\
\hline & $\mathrm{f}$ & $7.400 \pm 2.196$ & $8.425 \pm 1.931$ & $5.433 \pm 1.328$ & 0.454 \\
\hline & $\mathrm{i}$ & $2.161 \pm 1.066$ & $2.339 \pm 1.130$ & $2.199 \pm 0.994$ & 0.222 \\
\hline \multirow{6}{*}{ Angle $\left(^{\circ}\right)$} & HVA & $14.008 \pm 6.314$ & $15.989 \pm 6.244$ & $12.179 \pm 5.844$ & $0.001 *$ \\
\hline & IMA & $7.911 \pm 2.924$ & $8.224 \pm 3.002$ & $7.623 \pm 2.839$ & 0.211 \\
\hline & PASA & $5.244 \pm 3.472$ & $5.452 \pm 3.394$ & $5.812 \pm 3.275$ & 0.566 \\
\hline & DASA & $5.011 \pm 4.117$ & $5.554 \pm 4.466$ & $6.494 \pm 4.959$ & 0.17 \\
\hline & Meary & $18.779 \pm 11.841$ & $15.256 \pm 11.384$ & $24.545 \pm 10.303$ & $0.001 *$ \\
\hline & Pitch & $29.529 \pm 6.361$ & $30.644 \pm 6.194$ & $27.705 \pm 6.275$ & $0.001 *$ \\
\hline
\end{tabular}

$* P<0.001$ vs. left. Note: a total of 150 images (72 left, 78 right) have been used for this table. The mean long axis and short axis of each HB: a, b, c, d, e, and f; the mean distance between medial and lateral sesamoid bones: i; some mean angles on the first metatarsophalangeal joint: HVA, IMA, PASA, and DASA; the mean angel of instep: Meary and pitch.

TABLE 4: Mean values of measurements of sesamoid bones and the first metatarsophalangeal joint (mean \pm SD).

\begin{tabular}{|c|c|c|c|c|c|}
\hline & Term & Total & Male & Female & $P$ value \\
\hline \multirow{5}{*}{ Distance $(\mathrm{mm})$} & $\mathrm{a}$ & $11.765 \pm 1.905$ & $12.077 \pm 1.824$ & $11.161 \pm 1.932$ & $0.010 *$ \\
\hline & $\mathrm{b}$ & $8.198 \pm 1.354$ & $8.344 \pm 1.389$ & $7.888 \pm 1.116$ & $0.019 *$ \\
\hline & c & $11.882 \pm 1.361$ & $12.227 \pm 1.161$ & $11.276 \pm 1.508$ & $0.001 *$ \\
\hline & $\mathrm{d}$ & $8.948 \pm 1.132$ & $9.116 \pm 1.169$ & $8.561 \pm 0.971$ & $0.002 *$ \\
\hline & $\mathrm{i}$ & $2.161 \pm 1.066$ & $2.421 \pm 1.071$ & $1.756 \pm 0.992$ & $0.001 *$ \\
\hline \multirow{6}{*}{ Angle $\left({ }^{\circ}\right)$} & HVA & $14.008 \pm 6.314$ & $13.307 \pm 6087$ & $15.410 \pm 6.602$ & 0.062 \\
\hline & IMA & $7.911 \pm 2.924$ & $7.783 \pm 2.872$ & $8.168 \pm 3.038$ & 0.458 \\
\hline & PASA & $5.244 \pm 3.472$ & $6.053 \pm 3.297$ & $4.972 \pm 3.426$ & 0.217 \\
\hline & DASA & $5.011 \pm 4.117$ & $6.128 \pm 4.814$ & $5.096 \pm 4.177$ & 0.296 \\
\hline & Meary & $18.779 \pm 11.841$ & $18.274 \pm 12.348$ & $19.816 \pm 10.808$ & 0.375 \\
\hline & Pitch & $29.529 \pm 6.361$ & $29.864 \pm 6.183$ & $28.842 \pm 6.744$ & 0.621 \\
\hline
\end{tabular}

$* P<0.05$ vs. male. Note: a total of 150 images (100 male, 50 female) have been used for this table. The mean long axis and short axis of each HB: a, b, c, d, e, and $\mathrm{f}$; the mean distance between medial and lateral sesamoid bones: $\mathrm{i}$; some mean angles on the first metatarsophalangeal joint: HVA, IMA, PASA, and DASA; the mean angel of instep: Meary and pitch.

(13) Angle Meary: the first metatarsal angle of the talus

(14) Angle pitch: calcaneal inclination angle

Inclusion criteria are as follows: (1) the patients older than 18 years who had weight-bearing X-rays (AP and lateral) and CT of the same foot with both studies obtained of each other and (2) no history of the first metatarsophalangeal joint operation.

At the same time, the metatarsus had to be ruled out because of these situations: (1) trauma history of injury to the first metatarsophalangeal joint and (2) with osteoarthropathy of the first metatarsophalangeal joint, including osteoarthritis, tumor, and infection.
To ensure the evaluation of interobserver reliability, all subjects were carefully observed by the same investigator twice, who is a general orthopedic surgeon engaged in anatomical work for more than 5 years. Then, take the mean (accurate to $0.1 \mathrm{~mm}$ ).

2.4. Statistical Analysis. The parameters were expressed as the mean \pm standard deviation (SD). A statistical analysis was performed using SPSS, version 20.0 (IBM Corp., Armonk, NY, USA). The normality tests were used to check the variables studied, and the variables conformed to the normal distribution. Independent samples $t$-test was applied to identify differences between measurements in male patients and 
female patients. All hypothesis tests were implemented by adopting a 5\% significance level, and $P$ values equal to or smaller than 0.05 were considered statistically significant. Bivariate correlation tests the relationship between two variables shown by a simple scatter plot. $\alpha \leq 0.05$ is taken as the test level to tell if there is a significant correlation.

\section{Results}

In this study, a total of 14 qualitative features were evaluated on X-ray and 3D CT (by 3 trainees, respectively) among patients. All data were normally distributed. Meanwhile, the first metatarsophalangeal joint is divided into four different types: type I (no HSB, 1.3\%), type II (with one HSB, $0.07 \%$ ), type IIIa (with two HSBs when THB is bigger, $28 \%$ ), type IIIb (with two HSBs when FHB is bigger, $65.3 \%$ ), and type IV (with three HSBs, 4.7\%) (Figure 4). Regarding HVA in these types, there are 141 cases (94\%) with mild HV, 9 cases (6\%) with moderate HV, and no case (0\%) with severe HV. Regarding IMA in these types, there are 146 cases $(97.3 \%)$ with mild HV, 4 cases $(2.7 \%)$ with moderate HV, and no case (0\%) with severe HV (Table 1). Moreover, Table 2 lists the frequency of medial HSB displace grade found in 3D CT images. There are 46 (30.7\%) cases of Grade 0 dislocation, 57 (38\%) cases of Grade 1 dislocation, 43 (28.7\%) cases of Grade 2 dislocation, 43 (28.7\%) cases of Grade 3 dislocation, and 4 (2.7\%) cases of Grade 4 dislocation (Table 1). In Table 2, there are statistical differences between the dislocation and classification of the sesamoids $(P<0.05)$, which means type IIIa and type IIIb caught worse dislocation than other types, especially type IIIb.

The measurements of the length and angle on sesamoid bones and the first metatarsophalangeal joint are listed in Table 3 and Table 4 . Table 3 illustrates whether there are statistical differences between the left and right sides of all data, when Table 4 illustrates whether there are statistical differences between the male and female of all data. Among those, HVA, angle Meary, and angle pitch have statistical differences between left and right which are listed in Table 3 $(P<0.05)$. Also, all a, b, c, d, and i have statistical differences between male and female which are listed in Table 4 $(P<0.05)$. There is no statistical difference at angle between male and female. For qualitative assessments on $\mathrm{X}$-ray and CT, interreader reliability was good to excellent for all features (Table 5 and Table 6).

Comparing the relationship among HVA, IMA, and the classification of HSBs, HVA and IMA and HVA and the classification of HSBs have significant correlation. Type IIIb has a greater chance of catching HV than type I, type II, type IIIa, and type IV. These relationships are showed by a simple scatter plot and presented in Figures 5(a)-5(c).

\section{Discussion}

Thus far, physical examination, radiographs, and other specialized studies assist with the classification of sesamoid pathology $[7,8]$. And the main treatment about HV is surgery which includes tissue surgery and osseous surgery. At the beginning, most of the initial treatment involves an
TABLE 5: Interreader reliability on X-ray.

\begin{tabular}{lc}
\hline Measurements & $\begin{array}{c}\text { Kappa }(95 \%) \\
\text { *weighted kappa }\end{array}$ \\
\hline Hallux valgus angle & $0.92(0.88,0.96)$ \\
Intermetatarsal angle & $0.86(0.8,0.94)$ \\
Proximal articular set angle & $0.71(0.53,0.87)$ \\
Distal articular set angle & $0.67(0.58,0.86)$ \\
Angle Meary & $0.62(0.46,0.87)$ \\
Angle pitch & $0.67(0.46,0.82)$ \\
\hline
\end{tabular}

TABLE 6: Interreader reliability on CT.

\begin{tabular}{lc}
\hline Measurements & $\begin{array}{c}\text { Kappa (95\%) } \\
\text { *weighted kappa }\end{array}$ \\
\hline $\begin{array}{l}\text { The long axis of the medial distal sesamoid bone } \\
\text { The short axis of the medial distal sesamoid }\end{array}$ & $0.75(0.62,0.9)$ \\
$\begin{array}{l}\text { bone } \\
\text { The long axis of the lateral distal sesamoid bone }\end{array}$ & $0.71(0.65,0.82)$ \\
$\begin{array}{l}\text { The short axis of the lateral distal sesamoid } \\
\text { bone }\end{array}$ & $0.62(0.47,0.96)$ \\
$\begin{array}{l}\text { The long axis of the medial proximal sesamoid } \\
\text { bone }\end{array}$ & $0.77(0.62,0.96)$ \\
$\begin{array}{l}\text { The short axis of the medial proximal sesamoid } \\
\text { bone }\end{array}$ & $0.74(0.61,0.89)$ \\
$\begin{array}{l}\text { Distance between medial and lateral sesamoid } \\
\text { bones }\end{array}$ & $0.8(0.76,0.99)$ \\
Degree of dislocation in sesamoid bone & $1(1,1)$ \\
\hline
\end{tabular}

expectant treatment named accommodative orthosis, but surgical intervention has to be chosen in recalcitrant cases.

In this research, 150 metatarsals were measured by X-ray and three-dimensional (3D) computed tomographic (CT) reconstruction technique. The result can be seen that bipartite sesamoid bones are the largest number of types which covered $93.3 \%$. Among these, type IIIb (with two HSBs when FHB is bigger, 65.3\%) covered more than type IIIa (with two HSBs when THB is bigger, 28\%). Meanwhile, absence and three-seed bone also exist. Except that, the statistical analysis demonstrated that there is no statistical difference between left and right sides, except that HVA was $15.989 \pm 6.244$ $\mathrm{mm}$ on the left and $12.179 \pm 5.844 \mathrm{~mm}$ on the right $(P<0.001)$, Meary was $15.256 \pm 11.384 \mathrm{~mm}$ on the left and $24.545 \pm 10.303 \mathrm{~mm}$ on the right $(P<0.001)$, and pitch was $30.644 \pm 6.194 \mathrm{~mm}$ on the left and $27.705 \pm 6.275 \mathrm{~mm}$ on the right $(P<0.001)$. When the HVA on the left side is bigger than that on the right, bivariate correlation tests the relationship among HVA, IMA, and the classification of HSBs; the result demonstrated that HVA and IMA and HVA and the classification of HSBs have significant correlation, which means patients with bipartite sesamoid bones have a higher probability of HV and bigger HVA is accompanied by bigger IMA most of the time. Moreover, there are statistical differences that a was $12.077 \pm 1.824 \mathrm{~mm}$ on male and $11.161 \pm$ $1.932 \mathrm{~mm}$ on female $(P<0.05)$, b was $8.344 \pm 1.389 \mathrm{~mm}$ on male and $7.888 \pm 1.116 \mathrm{~mm}$ on female $(P<0.05)$, $\mathrm{c}$ was 


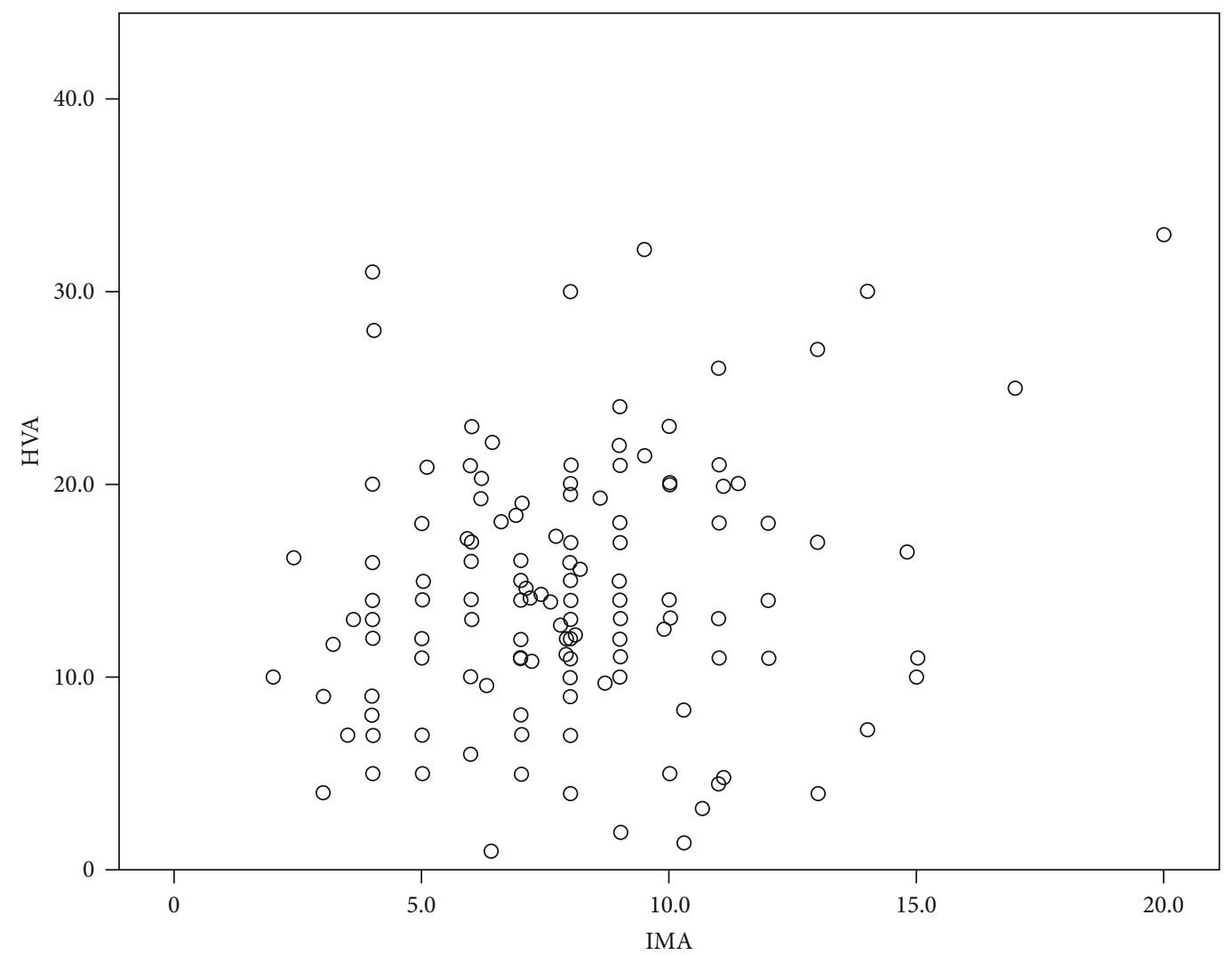

(a)

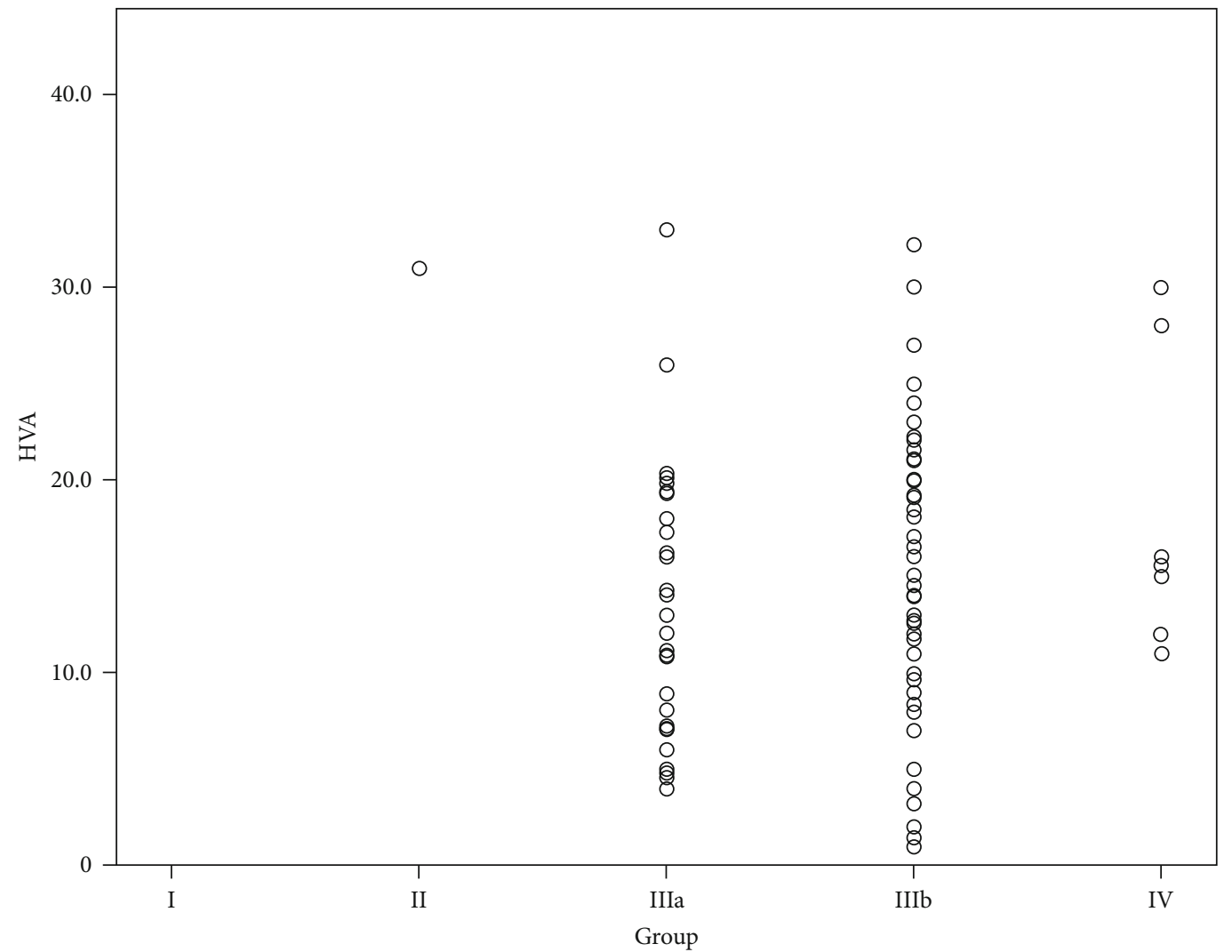

(b)

Figure 5: Continued. 


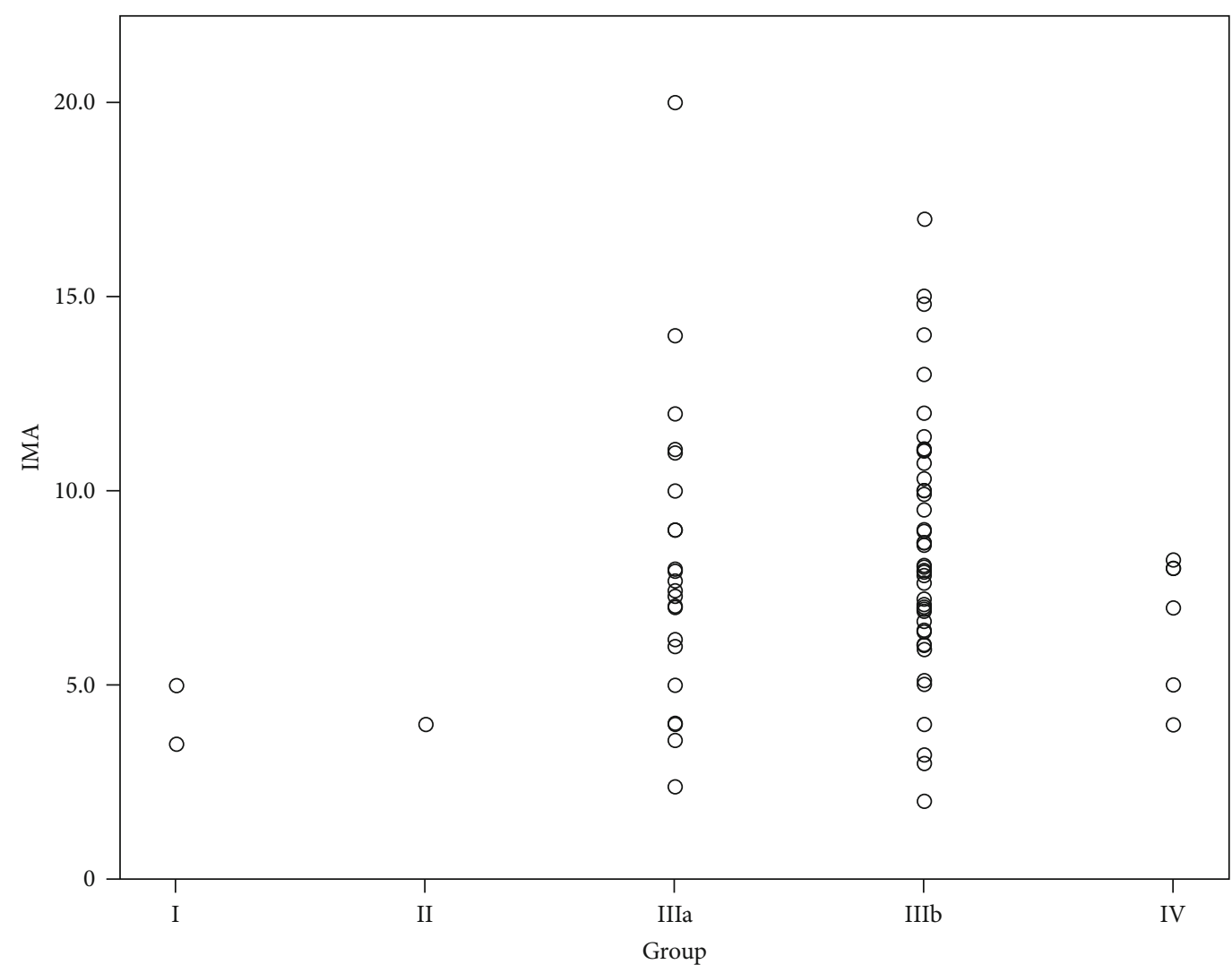

(c)

FIgURE 5: (a) Relationship between HVA and IMA. $* P<0.05, R=0.422$. Bigger HVA is accompanied by bigger IMA most of the time. (b) Relationship between HVA and the classification of HSBs. $* P<0.05, R=0.208$. Patients with type IIIa and type IIIb sesamoid bones have a higher probability of HV. (c) Relationship between IMA and the classification of HSBs. $* P>0.05, R=0.107$. There is no significant correlation between IMA and type group.

$12.227 \pm 1.161 \mathrm{~mm}$ on male and $11.276 \pm 1.508 \mathrm{~mm}$ on female $(P<0.05)$, d was $9.116 \pm 1.169 \mathrm{~mm}$ on male and $8.561 \pm 0.971 \mathrm{~mm}$ on female $(P<0.05)$, and $\mathrm{i}$ was $2.421 \pm$ $1.071 \mathrm{~mm}$ on male and $1.756 \pm 0.992 \mathrm{~mm}$ on female $(P<0.05)$. Some study pointed that the shape of the metatarsal head may be a cause of $\mathrm{HV}$ which means there are some differences about anatomic characteristics like those [19-21] such as the anatomic differences between type IIIa and type IIIb.

Moreover, when it comes to type, IIIa and IIIb caught worse dislocation than other types, especially type IIIb. The number of type may be a factor, the relationship between HVA and the classification of HSBs may be another. The latter can also be related to the anatomic structure. HV deformity consists of lateral deviation of the proximal phalanx on the metatarsal head with accompanying medial deviation of the first metatarsal which leaded to an enlargement of the medial bony eminence of the first metatarsal head [22-24]. As the deformity progresses, the medial bony eminence of the first metatarsal head can be accentuated with bursal thickening or inflammatory bursitis. Although pain will not occur to every patient and is not proportional to deformity, some soft tissues in osseous are prone to acute pollicis bursal inflammation caused by long-term compression and friction.
Long-term anomalies can lead to bias, oblique span, and even osteoarthritis which seriously affect the quality of life of patients which choose to seek medical treatment. Moreover, the injury of sesamoid includes sesamoid fracture (SF), sesamoid dislocation (SD), sesamoiditis, sesamoid osteomyelitis, and sesamoid tuberculosis. In summary, it is typically caused by a repetitive stress injury [2, 23-25].

In clinic, patients with sesamoid bone below the first metatarsal head were three to five times more likely to suffer $\mathrm{HV}$, and Chevron Akin Osteotomy is one of the most widely studied and widely used kinds of research [2-5, 26-31]. The measurement about 302 HSBs on 150 dry metatarsals from Chinese can help the clinician to master the basic anatomical structure and morphology of the Chinese HSBs, which can improve the anatomical data of it and be helpful in clinical diagnosis and treatment. Though there are many researches about $\mathrm{HV}$ or anatomic characteristic, hardly any research studied the relationship between them, including the classification.

Nevertheless, there are still some shortages. Firstly, though some classifications were listed here to help us do better in clinic, the sesamoid bones have a complex structure and the appearance of new type cannot be assured. Secondly, the samples in this research are limited to the southwest and 
the sample size is not so adequate now. Thirdly, the HSBs of some minors are not completely ossified and are not taken into account.

\section{Conclusion}

In summary, HVA and IMA and HVA and the classification of HSBs have a significant correlation. Type IIIb has a greater chance of catching HV than type I, type II, type IIIa, and type IV. Hence, the classification and location of HSBs can be an important basis to choose operation methods and provide data index for postoperation evaluation.

\section{Data Availability}

The related data used to support the findings of this study are restricted by the medical ethics committee of School of Basic Medical Sciences, Southwest Medical University. Data are available from Lei Zhang (email: zhanglei870722@126.com) for researchers who meet the criteria for access to confidential data.

\section{Ethical Approval}

All procedures were approved by the Medical Ethics Review Board of Affiliated Traditional Chinese Medicine Hospital of Southwest Medical University with the following reference number: KY2018012.

\section{Conflicts of Interest}

There was no conflict of interest that exists in the submission of this manuscript.

\section{Authors' Contributions}

Junqiu Wang, Jiaju Liu, and Jiangqin Luo contributed equally to this work. Junqiu Wang, Jiaju Liu, and Jiangqin Luo are the co-first authors. The manuscript was approved by all authors for publication.

\section{Acknowledgments}

This study was supported by the Technology Strategic Cooperation Project of Luzhou Municipal People's GovernmentSouthwest Medical University Science (2018LZXNYDZK43), the subitems of Talent Team Project of Academician Zhong Shizhen, Southwest Medical University, Luzhou Municipal People's Government (2018zszysrctdxm), and the Scientific Research Project of Affiliated Chinese Medicine Hospital of Southwest Medical University (National Natural Fund Training Project) (2019XYLH-001).

\section{References}

[1] Y. H. Park, C. D. Jeong, G. W. Choi, and H. J. Kim, "Effect of bipartite hallucal sesamoid on hallux valgus surgery," Foot \& Ankle International, vol. 38, no. 6, pp. 634-640, 2017.
[2] B. T. Dedmond, J. W. Cory, and A. McBryde Jr., "The hallucal sesamoid complex," The Journal of the American Academy of Orthopaedic Surgeons, vol. 14, no. 13, pp. 745-753, 2006.

[3] T. J. Holme, S. S. Sivaloganathan, B. Patel, and K. Kunasingam, "Third-generation minimally invasive chevron akin osteotomy for hallux valgus," Foot \& Ankle International, vol. 41, no. 1, pp. 50-56, 2020.

[4] G. B. Holmes Jr. and A. R. Hsu, "Correction of intermetatarsal angle in hallux valgus using small suture button device," Foot \& Ankle International, vol. 34, no. 4, pp. 543-549, 2013.

[5] K. Iliou, G. K. Paraskevas, P. Kanavaros et al., "Correlation of the hallux sesamoids' orientation with various anatomical parameters in patients with hallux valgus deformity," Cureus., vol. 11, no. 5, article e4643, 2019.

[6] N. H. Theumann, C. W. A. Pfirrmann, A. V. R. Mohana Borges, D. J. Trudell, and D. Resnick, "Metatarsophalangeal joint of the great toe: normal MR, MR arthrographic, and MR bursographic findings in cadavers," Journal of Computer Assisted Tomography, vol. 26, no. 5, pp. 829-838, 2002.

[7] R. A. Mann and M. J. Coughlin, "Hallux valgus and complications of hallux valgus," in Surgery of the Foot, R. A. Mann, Ed., pp. 65-1312, Mosby, St. Louis, 5th ed. edition, 1986.

[8] P. V. Munuera, G. Domínguez, M. Reina, and P. Trujillo, "Bipartite hallucal sesamoid bones: relationship with hallux valgus and metatarsal index," Skeletal Radiology., vol. 36, no. 11, pp. 1043-1050, 2007.

[9] J. Ferrari and J. Malone-Lee, "The shape of the metatarsal head as a cause of hallux abductovalgus," Foot \& Ankle International, vol. 23, no. 3, pp. 236-242, 2016.

[10] D. Fellner and P. B. Milsom, "Relationship between hallux valgus and first metatarsal head shape," British Journal of Podiatric Medicine, vol. 50, pp. 54-56, 1995.

[11] J. Hummel, J. Skweres, N. Heineman et al., "Hallux valgus assessment on X-ray and magnetic resonance imaging (MRI): correlation with qualitative soft tissue and internal derangement findings on MRI," European Journal of Radiology, vol. 113, pp. 24-31, 2019.

[12] J. L. Jones and J. M. Losito, "Tibial sesamoid fracture in a softball player," Journal of the American Podiatric Medical Association, vol. 97, no. 1, pp. 85-88, 2007.

[13] D. Karasick and K. L. Wapner, "Hallux valgus deformity: preoperative radiologic assessment," American Journal of Roentgenology, vol. 155, no. 1, pp. 119-123, 1990.

[14] T. H. Lui, K. B. Chan, and L. K. Chan, "Endoscopic distal softtissue release in the treatment of hallux valgus: a cadaveric study," Arthroscopy: The Journal of Arthroscopic \& Related Surgery, vol. 26, no. 8, pp. 1111-1116, 2010.

[15] C. Li, L. Lu, Y. Zhang, Q. C. Ai-Xin-Jue-Luo, Z. T. Wang, and J. F. Wang, "F-shaped osteotomy combined with basal opening wedge osteotomy for severe hallux valgus," Orthopaedic Surgery, vol. 11, no. 4, pp. 604-612, 2019.

[16] R. H. Hardy and J. C. Clapham, "Observations on hallux valgus; based on a controlled series," Journal of Bone and Joint Surgery. British Volume (London), vol. 33, pp. 376-391, 1951.

[17] R. W. Smith, J. C. Reynolds, and M. J. Stewart, "Hallux valgus assessment: report of research committee of American Orthopaedic Foot and Ankle Society," Foot \& Ankle, vol. 5, no. 2, pp. 92-103, 1984.

[18] D. Prieskorn, S. C. Graves, and R. A. Smith, "Morphometric analysis of the plantar plate apparatus of the first 
metatarsophalangeal joint," Foot \& Ankle, vol. 14, no. 4, pp. 204-207, 2016.

[19] P. R. D'Arcangelo, K. B. Landorf, S. E. Munteanu, G. V. Zammit, and H. B. Menz, "Radiographic correlates of hallux valgus severity in older people," Journal of Foot and Ankle Research, vol. 3, no. 1, 2010.

[20] S. Nix, M. Smith, and B. Vicenzino, "Prevalence of hallux valgus in the general population: a systematic review and metaanalysis," Journal of Foot and Ankle Research, vol. 3, no. 1, 2010.

[21] Z. Y. Chao and S. Z. Cheng, "Research progress on anatomy and biomechanics of the sesamoids below the head of the first metatarsal," Chinese Journal of Traumatology, vol. 24, no. 1, pp. 88-90, 2011.

[22] E. D. Sorene and G. Regev, "Complex dislocation with double sesamoid entrapment of the interphalangeal joint of the hallux," The Journal of Foot and Ankle Surgery, vol. 45, no. 6, pp. 413-416, 2006.

[23] A. M. McBryde Jr. and R. B. Anderson, "Sesamoid foot problems in the athlete," Clinics in Sports Medicine, vol. 7, no. 1, pp. 51-60, 1988.

[24] B. C. Min, C. Y. Chung, M. S. Park et al., "Dynamic first tarsometatarsal instability during gait evaluated by pedobarographic examination in patients with hallux valgus," Foot \& Ankle International, vol. 40, no. 9, pp. 1104-1109, 2019.

[25] A. MacMahon, J. Karbassi, J. C. Burket et al., "Return to sports and physical activities after the modified lapidus procedure for hallux valgus in young patients," Foot \& Ankle International, vol. 37, no. 4, pp. 378-385, 2016.

[26] W. Deng, Y. Chen, Y. X. Li et al., "Chevron osteotomy versus scarf osteotomy for the efficacy of radiographic and clinical in moderate and severe hallux valgus: a systematic review," Zhongguo Gu Shang, vol. 32, no. 8, pp. 765-771, 2019.

[27] H. J. Trnka, S. G. Hofstaetter, J. G. Hofstaetter, F. Gruber, S. B. Adams Jr., and M. E. Easley, "Intermediate-term results of the Ludloff osteotomy in one hundred and eleven feet," The Journal of Bone and Joint Surgery. American Volume, vol. 90, no. 3, pp. 531-539, 2008.

[28] L. S. Weil and M. Hill, "Bipartite tibial sesamoid and hallux abducto valgus deformity: a previously unreported correlation," The Journal of Foot Surgery, vol. 31, no. 2, pp. 104111, 1992.

[29] H. Waizy, M. Jäger, M. Abbara-Czardybon, T. G. Schmidt, and D. Frank, "Surgical treatment of AVN of the fibular (lateral) sesamoid," Foot and Ankle International., vol. 29, no. 2, pp. 231-236, 2008.

[30] D. Y. Wu and K. F. Lam, "Osteodesis for hallux valgus correction: is it effective?," Clinical Orthopaedics and Related Research, vol. 473, no. 1, pp. 328-336, 2015.

[31] D. Wai-Chi Wong, Y. Wang, M. Zhang, and A. Kam-Lun Leung, "Functional restoration and risk of non-union of the first metatarsocuneiform arthrodesis for hallux valgus: a finite element approach," Journal of Biomechanics, vol. 48, no. 12, pp. 3142-3148, 2015. 